\title{
ANALYTIC INVARIANTS OF BOUNDARY LINKS
}

\author{
STAVROS GAROUFALIDIS AND JEROME LEVINE
}

\begin{abstract}
Using basic topology and linear algebra, we define a plethora of invariants of boundary links whose values are power series with noncommuting variables. These turn out to be useful and elementary reformulations of an invariant originally defined by M. Farber [Fa2].
\end{abstract}

\section{INTRODUCTION}

1.1. History and Purpose. In a series of papers, M. Farber used homological methods to introduce an invariant of boundary links with values in a ring of rational functions with noncommuting variables [Fa2]. A similar invariant to that of Farber was recently introduced by V. Retakh, C. Reutenauer and A. Vaintrob [RRV] based on the notion of quasideterminants.

The purpose of this paper is to give an interpretation of Farber's invariant as a simple invariant of the Seifert matrix of a boundary link, which is more elementary and makes calculation more straightforward. From this point of view we will, in fact, define a whole spectrum of invariants which take values in noncommutative power series rings. Although these invariants all turn out to be determined by Farber's - see Theorem 2-it is useful to have the different formulations. An example is given by $\chi_{\Delta}$ - see page 3which has direct application to the study of the Kontsevich integral of a boundary link and its rationality properties, as will be explained in subsequent publications $[\mathrm{Ga}, \mathrm{GK}] . \chi_{\Delta}$ also gives a natural way to see that Farber's invariant determines the natural analog of the Alexander polynomial for a boundary link (the classical Alexander polynomial of a boundary link is 0). See Proposition 1.4.

We would like to thank Michael Farber for useful discussions.

1.2. Boundary links and their refinements. All manifolds will be oriented and all maps will be smooth and orientation preserving. A boundary link ( $\partial$-link) in a 3-manifold is an oriented link which is the boundary of a disjoint union of connected surfaces, each with one boundary component. A choice of such surfaces is called a Seifert surface of the boundary link. It is well-known that in the case of boundary links (unlike the case of knots) the cobordism class, relative boundary, of a Seifert surface for a given link is not unique. There are at least two ways to overcome this difficulty, as was explained by Cappell-Shaneson [CS] and Ko [K2]:

- A $\Sigma$-boundary link $L$ (or simply, a $\Sigma$-link) in a 3-manifold $M$ is a choice, up to isotopy, of Seifert surface $\Sigma$ in $S^{3}$ such that $\partial \Sigma=L$.

- An $F$-boundary link $L$ of $n$ components (or, simply, an $F$-link) is a link, up to isotopy, equipped with a map $\phi: \pi_{1}(M \backslash L) \rightarrow F$ where $F$ is the free group on $n$ letters and $\phi$ maps a choice of meridians of $L$ to a basis of $F$. $\phi$ is called a splitting map for $L$.

It turns out that $F$-links can be identified with the set of cobordism classes, rel boundary, (or tube equivalence classes) of Seifert surfaces - see Gutierrez and Smythe [Gu, Sm]. Let $A_{n}$ denote the group of automorphisms $\alpha$ of the free group $F\left(t_{1}, \ldots, t_{n}\right)$ that satisfy $\alpha\left(t_{i}\right)=w_{i} t_{i} w_{i}^{-1}$ for some $w_{i} \in F\left(t_{1}, \ldots, t_{n}\right)$, for all $i$, [CS, K2]. $A_{n}$ acts on the set of $F$-links by composition with the splitting map $\phi$. In [K2] a simple set of generators for $A_{n}$ was given, and the action of these generators was described geometrically as what

Date: This edition: October 19, 2000. First edition: October 17, 2000.

The authors were partially supported by NSF grants DMS-98-00703 and DMS-99-71802 respectively, and by an Israel-US BSF grant. This and related preprints can also be obtained at http://www.math.gatech.edu/ stavros and http://www.math.brandeis.edu/Faculty/jlevine/

1991 Mathematics Classification. Primary 57N10. Secondary 57M25.

Key words and phrases:Boundary links, Seifert matrices, S-equivalence. 
was there called cocooning. It turns out that the set of equivalences classes of $F$-links, modulo the $A_{n}$ action, can be identified with the set of $\partial$-links.

We denote by $X_{L}^{\omega}$ the $F$-covering of $S^{3}-L$ associated with $\operatorname{Ker} \phi=\pi_{\omega}$, the intersection of the lower central series of $\pi=\pi_{1}\left(S^{3}-L\right)$.

1.3. Seifert matrices of boundary links. There is an algebraic notion of a Seifert matrix associated to a $\Sigma$-link of $n$ components, [K1, K2]. These matrices are partitioned into $n \times n$ blocks of matrices, corresponding to the link components. Let $\operatorname{Sei}(n)$ denote the set of matrices $A=\left(A_{i j}\right)$ of square matrix blocks $A_{i j}$ for $i, j=1 \ldots, n$, with integer entries, satisfying the conditions

$$
A_{i j}^{\prime}=A_{j i} \text { for } i \neq j \text { and } \operatorname{det}\left(A_{i i}-A_{i i}^{\prime}\right)=1 \text { for all } i \text {. }
$$

Let Sei denote the set of all Seifert matrices. The Seifert matrix associated to a $\Sigma$-link (resp. $F$-link, $\partial$-link) is an element of $\operatorname{Sei}(n)$, well-defined up to $S_{1}$-equivalence (resp. $S_{12}$-equivalence, $S_{123}$ equivalence), where $S_{1}$ stands for congruence, $S_{2}$ stands for stabilization and $S_{3}$ stands for equivalence under an algebraic action of $A_{n}$ on Sei $(n)$ defined by Ko [K1, K2] (see Section 2.1 below). Note that $S_{123}=S_{12}$ for $n \leq 2$, since $A_{n}$ consists entirely of inner automorphisms which act trivially on Seifert matrices. We have a commutative diagram

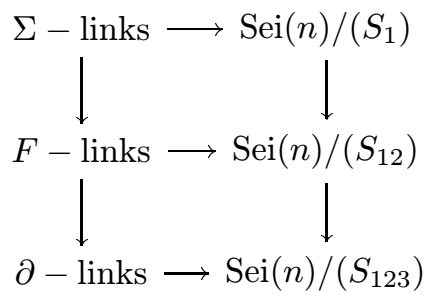

Set $\Lambda=\mathbb{Q}[F]$ the group-ring with rational coefficients and $\hat{\Lambda}$ its completion with respect to powers of the augmentation ideal. Then $A_{L}=H_{1}\left(X_{L}^{\omega}, \mathbb{Q}\right)$ is a $\Lambda$-module. Let $\Lambda^{\text {ab }}=\mathbb{Q}[H]$, where $H$ is the free abelian group on generators $\left(t_{1}, \ldots, t_{n}\right)$. If $X_{L}^{\mathrm{ab}}$ denotes the universal abelian covering of $S^{3}-L$, then $H_{1}\left(X_{L}^{\mathrm{ab}}, \mathbb{Q}\right)$ is a $\Lambda^{\mathrm{ab}}$-module. Note that $\hat{\Lambda}$ can be identified with the power series ring in the $n$ noncommuting variables $x_{i}=t_{i}-1$ and $\hat{\Lambda}^{\mathrm{ab}}$ with the power series ring in $n$ commuting variables $x_{i}=t_{i}-1 . \Lambda$ (and also, $\left.\hat{\Lambda}, \Lambda^{\mathrm{ab}}, \hat{\Lambda} \mathrm{ab}\right)$ are rings with (anti)-involution given by $g \rightarrow \bar{g}=g^{-1}$ for $g \in F$. Note that $\bar{x}_{i}=-\left(x_{i}+1\right)^{-1} x_{i}$. The action of $A_{n}$ on $F$ extends naturally to $\Lambda$ and $\hat{\Lambda}$ and induces the trivial action on $\Lambda^{\text {ab }}$. Now, we can introduce analytic invariants of the set Sei: Let $f \in \mathbb{Q}\langle\langle x, z\rangle\rangle$ be a noncommutative power series in two variables. We will say $f$ is admissible if, for any non-negative integer $n$, there are only a finite number of terms in $f$ of total $x$-degree $n$. The admissible power series form a subring $\mathcal{R}^{\text {ad }}$ of $\mathbb{Q}\langle\langle x, z\rangle\rangle$. Now let $X=\operatorname{diag}\left(x_{1}, \ldots, x_{n}\right)$ be a (block) diagonal matrix. Then we let

$$
\chi_{f}: \operatorname{Sei}(n) \rightarrow \hat{\Lambda} \text { be defined by } \chi_{f}(A)=\operatorname{tr}\left(f\left(X, Z_{A}\right)-f\left(X, I_{1 / 2}\right)\right)
$$

where $Z_{A}=A\left(A-A^{\prime}\right)^{-1}$ and $I_{1 / 2}$ is the block diagonal matrix in which half of the diagonal entries in each diagonal block are 0 and half are 1 . Note that $\left.f\left(X, I_{1 / 2}\right)\right)$ is independent of how the 0's and 1's are distributed

Theorem 1. For all admissible $f, \chi_{f}$ descends to a map

$$
\operatorname{Sei}(n) /\left(S_{12}\right) \rightarrow \hat{\Lambda}
$$

Remark 1.1. If $f \in \mathbb{Z}\langle\langle x, z\rangle\rangle$ then $\chi_{f}(A)$ has integer coefficients.

Question 1. If $\alpha \in A_{n}, f \in \mathcal{R}^{\text {ad }}$ and $A$ a Seifert matrix, is $\chi_{f}(\alpha \cdot A)$ determined by $\chi_{f}(A)$ and $\alpha$ ?

Let $\mathcal{R}\left(a_{1}, \ldots, a_{n}\right)$ denote the subring of $\mathbb{Q}\left\langle\left\langle a_{1}, \ldots, a_{n}\right\rangle\right\rangle$ consisting of the rational functions in the noncommuting variables $\left\{a_{1}, \ldots, a_{n}\right\}$ (see $[\mathrm{B}]$ ). This can be defined as the smallest subring of $\mathbb{Q}\left\langle\left\langle a_{1}, \ldots, a_{n}\right\rangle\right\rangle$ containing the polynomials $\mathbb{Q}\left[a_{1}, \ldots, a_{n}\right]$ and closed under the operation of taking inverses of special series, i.e. those $f$ with constant term $f(0, \ldots, 0)=1[\mathrm{~B}, \mathrm{p} .6]$. Let $\mathcal{R}_{*}(x, z)$ denote the smallest subring of $\mathcal{R}(x, z)$ containing the polynomials and closed under the operation of taking inverses of extra-special series, i.e. admissible $f(x, z)$ which satisfy $f(0, z)=1$. Clearly $\mathcal{R}_{*}(x, z) \subset \mathcal{R}(x, z)$. We also note that $\mathcal{R}_{*}(x, z) \subset \mathcal{R}^{\text {ad }}$ since it is not hard to see that if $f$ is special then $f^{-1}$ is admissible if and only $f$ is extra-special. 
Question 2. Is $\mathcal{R}_{*}(x, z)=\mathcal{R}(x, z) \cap \mathcal{R}^{\text {ad }}$ ?

Proposition 1.2. If $f(x, z) \in \mathcal{R}_{*}(x, z)$, then $\chi_{f} \in \mathcal{R}\left(x_{1}, \ldots, x_{n}\right)$.

$\chi_{f}$ satisfies a general duality property. Define anti-involutions $f \rightarrow \tilde{f}$ and $f \rightarrow \bar{f}$ on $\mathbb{Q}\langle\langle x, z\rangle\rangle$ and $\mathbb{Q}\left\langle\left\langle x_{1}, \ldots, x_{n}\right\rangle\right\rangle$ by the properties $\widetilde{x}=x, \bar{x}=-x(1+x)^{-1}, \widetilde{z}=\bar{z}=z, \widetilde{x}_{i}=x_{i}, \bar{x}_{i}=-x_{i}\left(1+x_{i}\right)^{-1}$ and $\widetilde{f g}=\widetilde{g} \tilde{f}, \overline{f g}=\bar{g} \bar{f}$, and an involution $f \rightarrow \hat{f}$ on $\mathbb{Q}\langle\langle x, z\rangle\rangle$ and $\mathbb{Q}\left\langle\left\langle x_{1}, \ldots, x_{n}\right\rangle\right\rangle$, by $\hat{x}=\bar{x}, \hat{z}=z, \hat{x}_{i}=\bar{x}_{i}$ and $\widehat{f g}=\hat{f} \hat{g}$. Note that the composition of any two of the maps $f \rightarrow \tilde{f}, \bar{f}$ or $\hat{f}$ on $\mathbb{Q}\left\langle\left\langle x_{1}, \ldots, x_{n}\right\rangle\right\rangle$ is equal to the third.

Proposition 1.3. For admissible $f$, we have that $\tilde{\chi}_{f(x, z)}=\chi_{\tilde{f}(x, 1-z)}$ and $\chi_{\hat{f}}=\hat{\chi}_{f}$. Therefore we also have $\bar{\chi}_{f(x, z)}=\chi_{\bar{f}(x, 1-z)}$.

Note that if $f$ is admissible then

- $\tilde{f}, \hat{f}$ are admissible, and

- $f(x, 1-z)$ is defined (which is not true for every $f \in \mathbb{Q}\langle\langle x, z\rangle\rangle)$ and admissible.

Let $f_{\Delta}=\log (x z+1) \in \mathbb{Q}\langle\langle x, z\rangle\rangle$. Note that $f_{\Delta}$ is admissible -in fact any $f \in \mathbb{Q}\langle\langle x, z\rangle\rangle$ of the form $f(x, z)=G(x z)$, where $G(y) \in \mathbb{Q}\langle\langle y\rangle\rangle$, is admissible. Let us denote $\chi_{f_{\Delta}}$ by $\chi_{\Delta}$. Our interest in $\chi_{\Delta}$ comes from the fact that it can be identified with the "wheels part" of a (version of) the Kontsevich integral of $F$-links, as will be explained in a separate publication, [GK]. For now, let us explain the relation between $\chi_{\Delta}$ and the algebraic topology of the complement of a boundary link $L$.

Let $\Delta^{b}(L) \in \Lambda^{\mathrm{ab}} /\left(\right.$ units), denote the order of the torsion $\Lambda^{\mathrm{ab}}$-module $A_{L}^{\mathrm{ab}}=\operatorname{torsion}_{\Lambda^{\mathrm{ab}}} H_{1}\left(X_{L}^{\mathrm{ab}}, \mathbb{Q}\right)$.

It is well-known that $\Delta^{b}=\Delta^{b}(L)$ satisfies:

1. $\Delta^{b}(1, \ldots, 1)= \pm 1$ and

2. $\Delta^{b}\left(t_{1}^{-1}, \ldots, t_{n}^{-1}\right)$ is a unit multiple of $\Delta^{b}\left(t_{1}, \ldots, t_{n}\right)$ in $\Lambda^{\mathrm{ab}}$.

It follows that we can choose a unique (normalized) representative in $\Lambda^{\text {ab }}$ such that

(1') $\Delta^{b}(1, \ldots, 1)=1$ and

(2') $\Delta^{b}\left(t_{1}^{-1}, \ldots, t_{n}^{-1}\right)=\Delta^{b}\left(t_{1}, \ldots, t_{n}\right)$.

We call this normalized representative the torsion polynomial of $L$.

Proposition 1.4. (a) (Abelianization) If $\chi_{\Delta}^{\mathrm{ab}}$ denotes the abelianization of $\chi_{\Delta}$, then

$$
\chi_{\Delta}^{\mathrm{ab}}=\log \Delta^{b} \in \hat{\Lambda}^{\mathrm{ab}} .
$$

where $\Delta^{b}$ is the torsion polynomial.

(b) (Realization) For every element $\lambda \in \Lambda$ with integer coefficients satisfying $\lambda(1, \ldots, 1)=1$ and $\lambda=\bar{\lambda}$, there exists an $F$-link $L$ with $H_{1}\left(X_{L}^{\omega}, \mathbb{Z}\right) \cong \Lambda /(\lambda)$, where $(\lambda)$ denotes the left ideal generated by $\lambda$. As a consequence every element $\Delta$ of $\Lambda^{\mathrm{ab}}$ satisfying (1') and (2') can be realized as the torsion polynomial of some boundary link.

(c) (Duality) $\chi_{\Delta}=\bar{\chi}_{\Delta}$ in $\hat{\Lambda} /$ (cyclic), the quotient of $\hat{\Lambda}$ by its subgroup generated by $(a b-b a)$, for $a, b \in \hat{\Lambda}$.

Thus, $\chi_{\Delta}^{\mathrm{ab}}$ determines the torsion polynomial. In contrast, the classical multivariable Alexander polynomial of a boundary link vanishes, and in general it is not known which Laurent polynomials can be realized as the multivariable Alexander polynomials of a link.

For an $F$-link $L$, we can think of $\chi_{\Delta}$ as an analogue of the order of the $\Lambda$-module $A_{L}$ (even though the notion of order does not make sense for $\Lambda$-modules).

Proposition 1.5. If $\Phi(x, z)=(x z+1)^{-1} x \in \mathbb{Q}\langle\langle x, z\rangle\rangle$, then $\chi_{\Phi}$ is related to Farber's $\chi$-function $[$ Fa2, Section 2.4] by the formula

$$
\chi-\chi_{\Phi}=\sum_{i=1}^{n} g_{i}\left(x_{i}-\bar{x}_{i}\right)
$$

for some non-negative integers $g_{i}$.

Remark 1.6. It follows from Farber's approach that $\chi$ only depends on the $\Lambda$-module $A_{L}$, and, therefore, this is also true for $\chi_{\Phi}$, when $\Phi(x, z)=(x z+1)^{-1} x$, since the integers $g_{i}$ are half the ranks of the $x_{i}$-components of the minimal lattice in $A_{L}$, as is demonstrated in the proof of the above proposition. 
Question 3. Is there some way to see directly that $\chi_{\Phi}$ depends only on $A_{L}$ ?

In [Fa2] it is shown that $\chi$ (and thus $\chi_{\Phi}$ ) satisfies the duality property $\chi+\bar{\chi}=0$. We reprove this using Proposition 1.5 and 1.3.

Proposition 1.7. For any F-link, we have

$$
\chi_{\Phi}=-\bar{\chi}_{\Phi}
$$

Question 4. (Realization) Can every rational power series $\rho$, with integer coefficients, satisfying the duality property $\rho=-\bar{\rho}$ be realized as $\chi_{\Phi}(L)$ for some $F$-link $L$ ?

Question 5. For the cyclic module in Proposition 1.4(c), what is $\chi$ ?

It is interesting, if perhaps disappointing, that this array of invariants are actually all determined by the original $\chi$ of Farber.

Theorem 2. For any $f \in \mathbb{Q}\langle\langle x, z\rangle\rangle, \chi_{f}(L)$ is completely determined by $\chi(L)$, and therefore depends only on $A_{L}$.

Remark 1.8. It is pointed out in [Fa2, Prop. 5.2] that $\chi(L)$ determines $A_{L}$ when it is semi-simple but not otherwise. For example, it follows from [Fa2, Prop. 2.5(c)] that $\chi$ is not sensitive to different extensions of the same modules. In particular, for a knot $K, \chi(K)$ is determined by the Alexander polynomial [Fa2, Section 10.4] and it is well-known that there exist knots with the same Alexander polynomial but different Alexander modules.

Finally we consider some examples. If $L$ is an $F$-link, let $L^{\prime}$ denote the reflection (sometimes called mirror image) of $L$ with the natural $F$-structure induced from that of $L$ by the automorphism of $F$ defined by $t_{i} \rightarrow t_{i}^{-1}$. If $A$ is a Seifert matrix of $L$ then $A^{\prime}$ is a Seifert matrix for $L^{\prime}$. Note that $Z_{A^{\prime}}=I-Z_{A}=S Z_{A}^{\prime} S^{-1}$.

Proposition 1.9. For any $f \in \mathbb{Q}\langle\langle x, z\rangle\rangle, \chi_{f}\left(L^{\prime}\right)=\tilde{\chi}_{\widetilde{f}}(L)$. In particular $\chi\left(L^{\prime}\right)=\tilde{\chi}(L)$.

For 2-component links we have not been able to find any examples such that $\chi\left(L^{\prime}\right) \neq \chi(L)$.

Question 6. Is $\tilde{\chi}(L)=\chi(L)$ for any 2-component $F$-link?

On the other hand for 3-component $F$-links it is not hard to find such examples.

Proposition 1.10. There exist 3-component F-links such that $\chi\left(L^{\prime}\right)=\widetilde{\chi}(L) \neq \chi(L)$.

\section{Proofs}

2.1. Proof of Theorem 1. Let us introduce three moves on the set Sei of Seifert matrices:

$S_{1}$ : Replace $A$ by $P A P^{\prime}$ for a block diagonal matrix $P=\operatorname{diag}\left(P_{1}, \ldots, P_{n}\right)$ of unimodular matrices $P_{i}$ with integer entries.

$S_{2}$ : Replace $A$ by

$$
\left(\begin{array}{ccc}
A & \rho & 0 \\
\rho^{\prime} & 0 & 1 \\
0 & 0 & 0
\end{array}\right) \quad \text { or }\left(\begin{array}{ccc}
A & \rho & 0 \\
\rho^{\prime} & 0 & 0 \\
0 & 1 & 0
\end{array}\right)
$$

for a column vector $\rho$, where, for some $i$, the two new rows are added to $A_{i j}, 1 \leq j \leq n$ and the two new columns are added to $A_{j i}, 1 \leq j \leq n$.

$S_{3}$ : The move that generates $A_{n}$-equivalence, where the algebraic action of $A_{n}$ on $\operatorname{Sei}(n)$ is described in $[\mathrm{K} 1, \mathrm{~K} 2]$.

Note that $S_{1}, S_{2}$ generate the so-called $S$-equivalence of Seifert matrices.

Given a Seifert matrix $A$, we define $Z_{A}=A\left(A-A^{\prime}\right)^{-1}$ and $S_{A}=A-A^{\prime}$ (or simply, $Z$ and $S$ in case $A$ is clear), following Seifert. Note that $S$ is block-diagonal. The behavior of $Z$ under $S$-equivalence of $A$ is described by the following elementary matrix calculation

Lemma 2.1. If $A \stackrel{S_{1}}{\rightsquigarrow} B=P A P^{\prime}$, then $Z_{B}=P Z_{A} P^{-1}$. If $A \stackrel{S_{2}}{\rightsquigarrow} B$, then

$$
Z_{B}=\left(\begin{array}{ccc}
Z_{A} & 0 & \star \\
\star & 1 & \star \\
0 & 0 & 0
\end{array}\right) \text { or }\left(\begin{array}{ccc}
Z_{A} & \star & 0 \\
0 & 0 & 0 \\
\star & \star & 1
\end{array}\right) \text {. }
$$


Proof of Theorem 1. We need to show that $\chi_{f}$ is invariant under the moves $S_{1}$ and $S_{2}$. If $A \stackrel{S_{1}}{\rightsquigarrow \text {, then }}$ $f\left(X, Z_{B}\right)=\operatorname{Pf}\left(X, Z_{A}\right) P^{-1}$ thus $\chi_{f}(B)=\chi_{f}(A)$. If $A \stackrel{S_{2}}{\rightsquigarrow} B$, then the following identity

$$
\left(\begin{array}{ccc}
C & 0 & \star \\
\star & c & \star \\
0 & 0 & 0
\end{array}\right)\left(\begin{array}{ccc}
C^{\prime} & 0 & \star \\
\star & c^{\prime} & \star \\
0 & 0 & 0
\end{array}\right)=\left(\begin{array}{ccc}
C C^{\prime} & 0 & \star \\
\star & c c^{\prime} & \star \\
0 & 0 & 0
\end{array}\right)
$$

implies that $\chi_{f}(B)=\chi_{f}(A)$.

Lemma 2.2. Given an $F$-link $L$ with a Seifert matrix $A$, then $X Z_{A}+I$ is a presentation matrix for $A_{L}$ over $\Lambda$, and for $A_{L}^{\mathrm{ab}}$ over $\Lambda^{a b}$.

Proof. It is well-known (see [K2]) that a presentation matrix for $A_{L}$ is $T A-A^{\prime}$, and similarly for $A_{L}^{\text {ab }}$. Since $T A-A^{\prime}=(T-I) A+\left(A-A^{\prime}\right)=(X Z+I)\left(A-A^{\prime}\right)$, the lemma follows.

2.2. Proof of Proposition 1.2. Let $\mathcal{R}^{\prime}(x, z)$ denote the subring of $\mathcal{R}^{\text {ad }}$ consisting of all admissible $f$ such that, for any scalar matrix $Z$ of the appropriate size, $f(X, Z)$ is a matrix all of whose entries are rational in $\mathbb{Q}\left\langle\left\langle x_{1}, \ldots, x_{n}\right\rangle\right\rangle$. It will suffice to show that $\mathcal{R}_{*}(x, z) \subset \mathcal{R}^{\prime}(x, z)$. To prove this we need to show that if $f \in \mathcal{R}^{\prime}(x, z)$ is extra-special, then $g=f^{-1} \in \mathcal{R}^{\prime}(x, z)$.

Consider the matrix equation $f(X, Z) g(X, Z)=I$. This defines a system of equations for the entries of $g(X, Z)$ of the form

$$
\sum_{j} a_{r j} y_{j}=b_{r}
$$

where $a_{r j}, b_{r} \in \mathcal{R}\left(x_{1}, \ldots, x_{n}\right)$. Since $f$ is extra-special, $f(0, Z)=I$, which implies that, with the correct choice of numbering of the equations, $a_{r j}(0, \ldots, 0)=\delta_{r j}$. Now we can apply [FV, Proposition 2.1] to conclude that the solutions $y_{r}$, which are the entries of $g(X, Z)$, are unique and rational.

2.3. Proof of Proposition 1.3. It follows from the definition of $Z$ that $Z+S Z^{\prime} S^{-1}=I$, where $S=A-A^{\prime}$. This implies that

$$
Z=S\left(I-Z^{\prime}\right) S^{-1}
$$

Thus

$$
\operatorname{tr} f(X, Z)=\operatorname{tr} f\left(X, I-Z^{\prime}\right)=\tilde{\operatorname{tr}} \tilde{f}(X, 1-Z)
$$

using the facts that $S$ commutes with $X$, that $\operatorname{tr} Y=\operatorname{tr} Y^{\prime}$, for any square matrix $Y$ and that $\operatorname{tr}(W Y)=$ $\operatorname{tr}(Y W)$, if the entries of $W$ commute with the entries of $Y$. From this we deduce the first equality.

The second equality is clear.

Remark 2.3. Let $\left[\mathcal{R}^{\text {ad }}, \mathcal{R}^{\text {ad }}\right]$ denote the abelian subgroup of the ring $\mathcal{R}^{\text {ad }}$ generated by $f g-g f$ for $f, g \in \mathcal{R}^{\text {ad }}$. It is easy to see that for all $f \in\left[\mathcal{R}^{\text {ad }}, \mathcal{R}^{\text {ad }}\right]$ we have $\chi_{f}=0 \in \hat{\Lambda} /$ (cyclic).

2.4. Proof of Proposition 1.4. To prove (a) first note that the normalized $\Delta^{b}$ can be defined by the equation

Thus we have

$$
\Delta^{b}=\operatorname{det}\left(T^{1 / 2} A-T^{-1 / 2} A^{\prime}\right)=\operatorname{det}\left((I+X)^{-1 / 2}(I+X Z)\right)
$$

$$
\begin{aligned}
\log \Delta^{b} & =\operatorname{tr} \log \left((I+X)^{-1 / 2}(I+X Z)\right) \\
& =\operatorname{tr} \log (I+X)^{-1 / 2}+\operatorname{tr} \log (I+X Z) \\
& =\operatorname{tr} \log (I+X Z)-\frac{1}{2} \operatorname{tr} \log (I+X)=\chi_{\Delta}
\end{aligned}
$$

This uses the following lemma, which is probably well-known.

Lemma 2.4. Suppose that $M, M_{1}, M_{2}$ are matrices of the form $I+N$ over a completed commutative power series ring, where $N$ has all entries of degree $>0$. Then we have the following identities.

$$
\begin{aligned}
\operatorname{tr} \log \left(M_{1} M_{2}\right) & =\operatorname{tr} \log \left(M_{1}\right)+\operatorname{tr} \log \left(M_{2}\right) \\
\log \operatorname{det}(M) & =\operatorname{tr} \log (M)
\end{aligned}
$$


Proof. (2) follows from the Campbell-Baker-Haussdorf formula and the fact that $\operatorname{tr}(A B)=\operatorname{tr}(B A)$ if $A, B$ are matrices over a commutative ring.

To prove (3), first note that it is obvious if $M$ is triangular. Secondly, it follows from (2) that if it is true for $M_{1}$ and $M_{2}$, then it is true for $M_{1} M_{2}$. Thus if will follow from the fact that any such $M$ can be written $M=L U$, where $L$ is lower triangular (i.e. $l_{i j}=0$ if $i<j$ ) and $U$ is upper triangular. We prove this by induction on the size of $M$.

Write $M=\left(\begin{array}{cc}u & \beta \\ \alpha & \widetilde{M}\end{array}\right)$, where $\alpha$ is a column vector and $\beta$ is a row vector. By induction we can write $\widetilde{M}-u^{-1} \alpha \beta=\widetilde{L} \widetilde{U}$, for triangular matrices $\widetilde{L}, \widetilde{U}$. Now we define

$$
L=\left(\begin{array}{cc}
u & 0 \\
\alpha & \widetilde{L}
\end{array}\right) \text { and } U=\left(\begin{array}{cc}
1 & u^{-1} \beta \\
0 & \widetilde{U}
\end{array}\right)
$$

One checks immediately that $M=L U$.

(b) follows from a general construction in [Le]. Consider the trivial link $L_{0} \subset S^{3}$ with $n$ components. Then the splitting map $\phi$ is an isomorphism. Consider the universal cover $X_{L_{0}}^{\omega}$ of $S^{3}-L_{0}$. Given $\lambda=\sum_{g \in F} a_{g} g$ satisfying $\lambda=\bar{\lambda}$, we can construct a simple closed curve $\gamma$ in $S^{3}-L_{0}$ which is null-homotopic and unknotted in $S^{3}$ such that, if $\widetilde{\gamma}$ is any lift of $\gamma$ in $X_{L_{0}}^{\omega}$ then the linking numbers of $\widetilde{\gamma}$ and its translates is given by

$$
\operatorname{lk}(\widetilde{\gamma}, g \widetilde{\gamma})=a_{g} \text { if } g \neq 1
$$

This construction is described in [Le]. Now do a +1 -surgery on $\gamma$ to produce $\Sigma^{3}$, which, since $\gamma$ was unknotted, is diffeomorphic to $S^{3}$. Let $L \subset S^{3}$ be the link corresponding to $L_{0} \subset \Sigma^{3}$ under such a diffeomorphism. Note that surgery on all the lifts of $\gamma$ produces an $F$-covering of $S^{3}-L$ and so $L$ is canonically a $F$-link. The argument in [Le] shows that $H_{1}\left(X_{L}^{\omega}\right) \cong \Lambda /(\lambda)$.

For (c), we will use Proposition 1.3 and Remark 2.3. Since $f_{\Delta}(x, z)=\log (1+x z)$, it is easy to see that $f_{\Delta}(x, z)=f_{\Delta}(z, x) \bmod \left[\mathcal{R}^{\text {ad }}, \mathcal{R}^{\text {ad }}\right]$. On the other hand, we have

$$
\begin{aligned}
\tilde{f}_{\Delta}(x, 1-z) & =\log (1+(1-z) x)=\log (1+x-z x)=\log ((1+z \bar{x})(1+x)) \\
& =\log (1+z \bar{x})+\log (1+x) \bmod \left[\mathcal{R}^{\mathrm{ad}}, \mathcal{R}^{\mathrm{ad}}\right] \\
& =\bar{f}_{\Delta}(x, z)+\log (1+x) \bmod \left[\mathcal{R}^{\mathrm{ad}}, \mathcal{R}^{\mathrm{ad}}\right] \\
& =\log (1+\bar{x} z)+\log (1+x) \bmod \left[\mathcal{R}^{\mathrm{ad}}, \mathcal{R}^{\mathrm{ad}}\right] \\
& =\hat{f}_{\Delta}(x, z)+\log (1+x) \bmod \left[\mathcal{R}^{\mathrm{ad}}, \mathcal{R}^{\mathrm{ad}}\right] .
\end{aligned}
$$

Proposition 1.3 and Remark 2.3 imply that

$$
\chi_{f_{\Delta}(x, z)}=\tilde{\chi}_{\widetilde{f}_{\Delta}(x, 1-z)}=\tilde{\chi}_{\hat{f}_{\Delta}(x, z)}+\chi_{\log (1+x)}=\bar{\chi}_{f_{\Delta}}+\chi_{\log (1+x)} \in \hat{\Lambda} /(\text { cyclic }) .
$$

Since $\chi_{\Delta}\left(x_{i}\right)=\chi_{f_{\Delta}(x, z)}$ and $\chi_{\log (1+x)}=0$, it follows that $\chi_{\Delta}=\bar{\chi}_{\Delta} \in \hat{\Lambda} /($ cyclic) .

2.5. Proof of Proposition 1.5. Let $A$ be any Seifert matrix for $L$. We can construct a higher-dimensional simple link $\widetilde{L}$ in $S^{4 k+3}$, for some large $k$, which has a Seifert manifold $W$ yielding $A$ as its Seifert matrix (see, e.g. [K2]). Since the Seifert matrix determines the link module, via the presentation matrix $T A-A^{\prime}$ we have $A_{L}=H_{1}\left(X_{L}^{\omega}, \mathbb{Q}\right) \cong A_{\widetilde{L}}=H_{2 k+1}\left(X_{\widetilde{L}}^{\omega}, \mathbb{Q}\right)$. Therefore $\chi$ for $A_{L}$ is the same as $\chi$ for $A_{\widetilde{L}}$. Now we can do surgery on $W$ to obtain a minimal Seifert manifold $V$ for $\widetilde{L}$, whose components are $2 k$-connected-see [Fa1, Section 6.12] and [Gu]. This determines a minimal lattice $J$ for $A_{\widetilde{L}}$, according to [Fa2, p.563-4]. The Seifert matrix $B$ determined by $V$ is S-equivalent to $A$ and so $\chi_{\Phi}(L)=\operatorname{tr}\left((I+X Z)^{-1} X\right)-\operatorname{tr}\left(\left(I+X I_{1 / 2}\right)^{-1} X\right)$, where $Z=B\left(B-B^{\prime}\right)^{-1}$. Note that $J=\bigoplus_{i} x_{i} J$ and each $x_{i} J$ is isomorphic to $H_{2 q+1}\left(V_{i}\right)$, where $V_{i}$ is the $i$-th component of $V$. Then, if $2 g_{i}=\operatorname{rank} H_{2 q+1}\left(V_{i}\right)$, it is straightforward to check that $\operatorname{tr}\left(\left(I+X I_{1 / 2}\right)^{-1} X\right)=$ $\sum_{i} g_{i}\left(x_{i}-\bar{x}_{i}\right)$. The proof will be completed if we show that $\chi=\operatorname{tr}\left((I+X Z)^{-1} X\right)$.

Now $A_{L}$ is the $\Lambda$-module with presentation matrix $X Z+I$, as in Lemma 2.2. The generators $\alpha_{r}$ of $A_{L}$, corresponding to the columns of $X Z+I$, span the minimal lattice $J$ as described in [Fa2, p.564], since $B$ comes from a minimal Seifert manifold. If we let $M_{i}=x_{i} A_{L}$, then the generators corresponding to the $i$ th column block of $X Z+I$ generate $M_{i}$ since, if $\alpha_{r}$ denotes a generator corresponding to a column in the $i$ th column block, the $r$-th row of $X Z+I$ gives the relation $\alpha_{r}=-x_{i} \sum Z_{r s} \alpha_{s}$. Thus, $\pi_{i}$ is given by the matrix 
$P_{i}=\left(\begin{array}{ccc}0 & & \\ & I & \\ & & 0\end{array}\right)$ where $I$ is in the $(i, i)$ block, $z$ is given by the matrix $Z^{\prime}$ and $\partial_{i}$ is given by the matrix whose $i$ th column block is the $i$ th column block of $-Z^{\prime}$ and the other columns are zero-call this matrix $Z_{i}$. Now, $\chi$ is given by

$$
\chi=\sum_{k} \sum_{n} \operatorname{tr}\left(\pi_{k} \partial_{a_{1}} \cdots \partial_{a_{n}}\right) x_{a_{n}} \cdots x_{a_{1}} x_{k}
$$

But $\pi_{k} \partial_{a_{1}} \cdots \partial_{a_{n}}$ is given by the matrix $P_{k} Z_{a_{1}}^{\prime} \cdots Z_{a_{n}}^{\prime}$. Note that $Z_{a_{1}}^{\prime} \cdots Z_{a_{n}}^{\prime}$ is the matrix with only the $a_{n^{-}}$ th column block nonzero and the $\left(r, a_{n}\right)$ block is $(-1)^{n} Z_{r, a_{1}}^{\prime} Z_{a_{1}, a_{2}}^{\prime} \cdots Z_{a_{n-1}, a_{n}}^{\prime}$. Multiply this by $P_{k}$, giving a matrix whose only nonzero entries are in the $\left(k, a_{n}\right)$ block, and equal to $(-1)^{n} Z_{k, a_{1}}^{\prime} Z_{a_{1}, a_{2}}^{\prime} \cdots Z_{a_{n-1}, a_{n}}^{\prime}$. Thus, we have a nonzero trace only if $k=a_{n}$, giving

$$
\begin{aligned}
\chi & =\sum_{n} \sum_{a_{1}, \ldots, a_{n}}(-1)^{n} \operatorname{tr}\left(Z_{a_{n}, a_{1}}^{\prime} Z_{a_{1}, a_{2}}^{\prime} \cdots Z_{a_{n-1}, a_{n}}^{\prime}\right) x_{a_{n}} \cdots x_{a_{1}} x_{a_{n}} \\
& =\sum_{n} \sum_{a_{1}, \ldots, a_{n}}(-1)^{n} \operatorname{tr}\left(Z_{a_{n}, a_{n-1}} Z_{a_{n-1}, a_{n-2}} \cdots Z_{a_{1}, a_{n}}\right) x_{a_{n}} \cdots x_{a_{1}} x_{a_{n}} \\
& =\sum_{n} \sum_{a_{1}, \ldots, a_{n}, a_{n}^{\prime}}(-1)^{n} \operatorname{tr}\left((X Z)_{a_{n}, a_{n-1}}(X Z)_{a_{n-1}, a_{n-2}} \cdots(X Z)_{a_{1}, a_{n}^{\prime}} X_{a_{n}^{\prime}, a_{n}}\right) \\
& =\sum_{n}(-1)^{n} \operatorname{tr}\left((X Z)^{n} X\right)=\operatorname{tr}\left((X Z+I)^{-1} X\right) .
\end{aligned}
$$

2.6. Proof of Proposition 1.7. It is easy to see that $\widetilde{\Phi}(x, z)=\Phi(x, z)$. Furthermore, $\operatorname{since} \operatorname{tr} \Phi\left(X, I_{1 / 2}\right)$ satisfies the asserted duality statements, we can omit this part of the definition of $\chi_{\Phi}$ in the following. We have:

$$
\begin{aligned}
\widetilde{\Phi}(x, 1-z) & =(1+x(1-z))^{-1} x=(1+x-x z)^{-1} x=(1+\bar{x} z)^{-1}(1+x)^{-1} x \\
& =-(1+\bar{x} z)^{-1} \bar{x}=-\Phi(\bar{x}, z)=-\hat{\Phi}(x, z) .
\end{aligned}
$$

Proposition 1.3 implies that $\chi_{\Phi}=\widetilde{\chi}_{\widetilde{\Phi}(x, 1-z)}=-\widetilde{\chi}_{\hat{\Phi}}=-\tilde{\hat{\chi}}_{\Phi}=-\bar{\chi}_{\Phi}$.

2.7. Proof of Theorem 2. It suffices to consider the case where $f$ is a monomial, say

$$
f=x^{f_{0}} z^{e_{1}} x^{f_{1}} \cdots z^{e_{k}} x^{f_{k}}
$$

where $e_{i}>0$ for $1 \leq i \leq k$ and $f_{i}>0$ if $0<i<k$. Note that we have a general formula

$$
\operatorname{tr} f(X, Z)=\sum_{i_{1}, \ldots, i_{k}} \operatorname{tr}\left(Z^{e_{1}}\right)_{i_{1} i_{2}}\left(Z^{e_{2}}\right)_{i_{2} i_{3}} \cdots\left(Z^{e_{k}}\right)_{i_{k} i_{1}} x_{i_{1}}^{f_{0}} x_{i_{2}}^{f_{1}} \cdots x_{i_{k}}^{f_{k-1}} x_{i_{1}}^{f_{k}}
$$

where $\left(Z^{e}\right)_{i j}$ denote the $(i, j)$-block of $Z^{e}$. Now we associate with $f$ another monomial $f^{\prime} \in \mathbb{Q}\langle\langle x, y, z\rangle\rangle$ by replacing each $z^{e_{i}}$ in $f$ by $(z y)^{e_{i}-1} z$, for every $1 \leq i \leq k$ and replacing each $x^{f_{i}}$ by $x$, for every $0 \leq i \leq k$ (even when $f_{0}$ or $f_{k}$ is zero). Now consider $\operatorname{tr} f^{\prime}(X, Y, Z)$, where $Y=\operatorname{diag}\left(y_{1}, \ldots, y_{n}\right)$ is a matrix identical to $X$ in which each $x_{i}$ is replaced by a new variable $y_{i}$. It is not hard to see, using equation (4), that $\operatorname{tr} f^{\prime}(X, Y, Z)$ and $f$ determine $\operatorname{tr} f(X, Z)$ by replacing each $x_{j}$ in $\operatorname{tr} f^{\prime}(X, Y, Z)$ with the appropriate power of $x_{j}$ and each $y_{j}$ by 1 . Furthermore, again using equation (4), $\operatorname{tr} f^{\prime}(X, X, Z)$ and $f \operatorname{determine} \operatorname{tr} f^{\prime}(X, Y, Z)$ since $f$ tells us which $x_{i}$ in $f^{\prime}(X, X, Z)$ to replace by $y_{i}$ to obtain $\operatorname{tr} f^{\prime}(X, Y, Z)$.

Finally we note that $f^{\prime}(x, x, z)$ is a monomial of the form $(x z)^{k} x$, and so coincides, up to sign, with the degree $k+1$ part of $\Phi$. Thus $\operatorname{tr} f^{\prime}(X, X, Z)$ is determined by $\operatorname{tr} \Phi(X, Z)$. This completes the proof.

2.8. Proof of Propositions 1.9 and 1.10. Since $\operatorname{tr} f\left(X, S Z^{\prime} S^{-1}\right)=\operatorname{tr} f\left(X, Z^{\prime}\right)=\widetilde{\operatorname{tr}} \tilde{f}(X, Z)$, we conclude that $\chi_{f}\left(L^{\prime}\right)=\widetilde{\chi}_{\widetilde{f}}(L)$. Since $\widetilde{\Phi}=\Phi$, it follows from Proposition 1.5 that $\chi\left(L^{\prime}\right)=\widetilde{\chi}(L)$. This proves Proposition 1.9.

For Proposition 1.10 let us consider the matrix

$$
A=\left(\begin{array}{rrr}
M & -S & -S \\
S & M & -S \\
S & S & M
\end{array}\right)
$$


where $S=\left(\begin{array}{rr}0 & 1 \\ -1 & 0\end{array}\right)$ and $M$ is any $2 \times 2$-matrix satisfying $M-M^{\prime}=S$. This is a Seifert matrix for some $F$-link $L$.

Then

$$
Z_{A}=\left(\begin{array}{rrr}
N & -I & -I \\
I & N & -I \\
I & I & N
\end{array}\right)
$$

where $N=M S$. From the general formula

$$
\operatorname{tr}_{\Phi}(Z)=\sum \operatorname{tr} x_{i_{1}} Z_{i_{1} i_{2}} x_{i_{2}} \cdots x_{i_{k}} Z_{i_{k} i_{1}} x_{i_{1}}=\sum\left(\operatorname{tr} Z_{i_{1} i_{2}} \cdots Z_{i_{k} i_{1}}\right) x_{i_{1}} x_{i_{2}} \cdots x_{i_{k}} x_{i_{1}}
$$

which follows from equation (4), we can see that $\operatorname{tr}_{\Phi}\left(Z_{A}\right)=\sum_{\mathbf{m}} a_{\mathbf{m}} \mathbf{m}$, summing over non-commutative monomials $\mathbf{m}=x_{i_{1}} x_{i_{2}} \cdots x_{i_{k}} x_{i_{1}}$, where $a_{\mathbf{m}}=(-1)^{r} \operatorname{tr} M^{s}$ with $r=\#\left\{j: i_{j+1}>i_{j}\right\}$ and $s=\#\left\{j: i_{j}=\right.$ $\left.i_{j+1}\right\}$. Thus, for example if $\mathbf{m}=x_{1} x_{2} x_{3} x_{1}$ then $a_{\mathbf{m}}=2$ whereas if $\mathbf{m}=x_{1} x_{3} x_{2} x_{1}$ then $a_{\mathbf{m}}=-2$. Thus $\chi_{\Phi}(A) \neq \widetilde{\chi}_{\Phi}(A)$ and we have the desired example.

\section{REFERENCES}

[B] J. Berstel and C. Reutenauer, Rational SERIES AND their LAnguages, EATCS Monographs on theoretical computer science 12, Springer-Verlag (1984).

[CS] S. Cappell and J. Shaneson, Link cobordism, Commentarii Math. Helv. 55 (1980) 20-49.

[Du] M. Duval, Forme de Blanchfield et cobordism d'enterlacs bords, Comment. Math. Helv. 61 (1986) 617-635.

[Fa1] M. Farber, Hermitian forms on link modules, Comment. Math. Helv. 66 (1991) 189-236.

[Fa2] - Noncommutative rational functions and boundary links, Math. Annalen 293 (1992) 543-568.

[FV] and P. Vogel, The Cohn localization of the free group ring, Math. Proc. Cambridge Phil. Soc. 111 (1992) $432-443$.

[GK] S. Garoufalidis and A. Kricker, Boundary links, localization and the Kontsevich integral, in preparation.

[Ga] Boundary links, localization and the loop move, in preparation.

[Gu M. Gutierrez, Boundary links and an unlinking theorem, Trans. Amer. Math. Soc. 171 (1972) 491-499.

[K1] K.H. Ko, Seifert matrices and boundary links, thesis, Brandeis University, 1984.

[K2] Seifert matrices and boundary link cobordisms, Trans. Amer. Math. Soc. 299 (1987) 657-681.

[Le] J. Levine, A method of generating link polynomials, Amer. J. Math., 89 (1967), 69-84.

[RRV] V. Retakh, C. Reutenauer and A. Vaintrob, Noncommutative rational functions and Farber's invariants of boundary links, AMS Transl. 194 (1999) 237-245.

[Sm] N. Smythe, Boundary links, Topology Seminar (Wisconsin 1965) Princeton Univ. Press, Princeton, NJ 1966, 69-72.

School of Mathematics, Georgia Institute of Technology, Atlanta, GA 30332-0160, USA.

E-mail address: stavros@math.gatech.edu

Department of Mathematics, Brandeis University, Waltham, MA 02454-9110, USA.

E-mail address: levine@brandeis.edu 